\title{
Unexpected effects of thickness and strain on superconductivity and magnetism in optimally doped $\mathrm{La}_{1.84} \mathrm{Sr}_{0.16} \mathrm{CuO}_{4}$ thin films
}

\author{
L. Howald, ${ }^{1,2,3,{ }^{*}}$ E. Stilp,,${ }^{1,4}$ F. Baiutti, ${ }^{6}$ C. Dietl, ${ }^{6}$ F. Wrobel, ${ }^{6}$ G. Logvenov, ${ }^{6}$ T. Prokscha, ${ }^{4}$ Z. Salman, ${ }^{4}$ \\ N. Wooding, ${ }^{7}$ D. Pavuna, ${ }^{7}$ H. Keller, ${ }^{1}$ and A. Suter ${ }^{4}$ \\ ${ }^{1}$ Physik-Institut der Universität Zürich, Winterthurerstrasse 190, CH-8057 Zürich, Switzerland \\ ${ }^{2}$ Swiss Light Source, Paul Scherrer Institut, CH-5232 Villigen PSI, Switzerland \\ ${ }^{3}$ Hochalpines Institut Ftan AG, CH-7551 Ftan, Switzerland \\ ${ }^{4}$ Laboratory for Muon Spin Spectroscopy, Paul Scherrer Institut, CH-5232 Villigen PSI, Switzerland \\ ${ }^{5}$ Materials for Energy Conversion, Empa, CH-8600 Dübendorf, Switzerland \\ ${ }^{6}$ Max Planck Institute for Solid State Research, Heisenbergstr. 1, D-70569 Stuttgart, Germany \\ ${ }^{7}$ Institute of Physics of Complex Matter, École Polytechnique Fédérale de Lausanne (EPFL), CH-1015 Lausanne, Switzerland
}

(Received 4 July 2017; revised manuscript received 28 January 2018; published 26 March 2018)

\begin{abstract}
The magnetic field distribution of the vortex lattice of optimally doped $\mathrm{La}_{1.84} \mathrm{Sr}_{0.16} \mathrm{CuO}_{4}$ thin films of various thicknesses, grown on different substrates, was investigated. The influence of film thickness and biaxial strain on the magnetic penetration depth and the superconducting number density were studied using muon spin rotation and compared to single-crystal results. We found an effective superconducting layer thickness smaller than the total film thickness, implying that the interfaces of the films are not or less superconducting than the bulk of the film. The superfluid density diminished in thinner films whereas compressive strain enhanced it. This shows that the number density of superconducting carriers is strongly affected by the boundary conditions as well as by the strain. Furthermore, in fully relaxed optimally doped $\mathrm{La}_{1.84} \mathrm{Sr}_{0.16} \mathrm{CuO}_{4}$ films grown on $\mathrm{SrTiO}_{3}$, we found a low-temperature magnetic state which sets in at $T_{c}$. It is reasoned that defects at the surface slow down high-frequency magnetic fluctuations such that a "quasistatic" magnetic ground state results, which coexists with the diminished surface superconductivity. These results indicate that the properties of the surface of optimally doped $\mathrm{La}_{1.84} \mathrm{Sr}_{0.16} \mathrm{CuO}_{4}$ superconductors differ substantially from the bulk.
\end{abstract}

DOI: 10.1103/PhysRevB.97.094514

\section{INTRODUCTION}

Since the discovery of high-temperature superconductivity [1], understanding the phase diagram, and especially the role of tuning parameters on superconductivity, has been the subject of interest for many studies. In $\mathrm{La}_{2-x} \mathrm{Sr}_{x} \mathrm{CuO}_{4+\epsilon}$ the critical temperature $\left(T_{c}\right)$ can be tuned by different techniques such as chemical doping [2], hydrostatic pressure [3], or biaxial pressure $[4,5]$. The variation of $T_{c}$ can be ascribed either to a modification of the carrier concentration or to a modification of the amplitude of the pairing interaction. Disentangling these effects is one of the important challenges to understand the mechanism of unconventional superconductivity.

The magnetic penetration depth $\lambda$ offers a direct probe to the mechanism leading to the variation of $T_{c}$. Indeed, within the London model

$$
\lambda=\sqrt{\frac{m^{\star} c^{2}}{4 \pi n_{S} e}} .
$$

$\lambda$ is directly related to the density of superconducting carriers, known as the number density $n_{S}$, and to the effective mass $m^{\star}$. Here, $e$ is the quasiparticle (electron/hole) charge and $c$ is the speed of light. The mass renormalization directly depends on the interactions affecting the quasiparticles, notably the

*ludovic.howald@gmail.com attracting interaction leading to superconductivity. The number density for an invariant gap symmetry is proportional to the carrier concentration. Therefore, within a given phase diagram, one expects the dependence of the superconducting transition temperature on the magnetic penetration depth to have a positive derivative $d T_{c}(\lambda) / d \lambda>0$ if the phase diagram is mainly determined by a variation of pairing interaction and a negative derivative $d T_{c}(\lambda) / d \lambda<0$ if the phase diagram is mainly determined by a variation of the carrier concentration [6].

Superconductivity is defined by the absence of electrical resistivity and expulsion of the magnetic field (Meissner effect). The second criterion implies that superconductivity is a bulk phase, as a certain thickness is required for superconducting currents to flow and expel the externally applied magnetic field. On the other hand, the superconducting wave function is extended and Cooper pairs can tunnel through nonsuperconducting regions such as in Josephson junctions [7]. Surface-sensitive techniques such as angle-resolved photoemission spectroscopy (see, e.g., [8]) have claimed important results on the superconducting phase, notably its single band electronic structure and potential relation to other orders such as magnetism. However, a proper understanding of these experiments is only possible once the relation between surface and bulk properties is established.

The application of in-plane compressive or tensile strain in thin films has a tremendous effect on superconductivity. In the cuprate system $\mathrm{La}_{2-x} \mathrm{Sr}_{x} \mathrm{CuO}_{4+\epsilon}$, biaxial compressive strain increases $T_{c}$ up to a factor 2 (see Ref. [9]) while biaxial tensile 
strain reduces every feature of superconductivity up to a complete suppression [5,10,11]. To disentangle possible origins to the modification of $T_{c}$, we present a systematic study of the magnetic penetration depth on thin-film $\mathrm{La}_{1.84} \mathrm{Sr}_{0.16} \mathrm{CuO}_{4}$ (LSCO) samples of different thicknesses grown on different substrates. The results are compared to single-crystal measurements. The muon spin rotation $(\mu \mathrm{SR})$ technique was used as it allows an absolute measurement of $\lambda$, and at the same time $\mu$ SR can detect even extremely weak local magnetic ground states. The local probe character of the muon technique is used to discriminate surface and bulk properties.

In thin films, using the $\mu \mathrm{SR}$ technique, the magnetic penetration depth is typically determined by probing the depth profile of an externally applied magnetic field parallel to the film surface in the Meissner state [12]. In the present case, extremely thin films (thickness $d \ll \lambda$ ) are investigated in order to preserve the biaxial strain induced by the substrate. Under this condition, the field variation in the Meissner state is too small to be resolved experimentally. Additionally, the muon implantation profile is too broad to precisely determine the magnetic depth profile. Therefore, we probed the magnetic field distribution induced by the vortex lattice in the mixed state, with a magnetic field perpendicular to the film surfaces, as is done in the bulk case [13]. However, the vortex state in a film of thickness $d \ll \lambda$ is different compared to the bulk since the vortices expand towards the surface [14]. In order to precisely understand this effect and extract the intrinsic magnetic penetration depth of the material under biaxial strain, we probed the magnetic field dependence of the field distribution due to the vortex lattice.

\section{EXPERIMENTAL DETAILS}

The $\mathrm{La}_{1.84} \mathrm{Sr}_{0.16} \mathrm{CuO}_{4}$ epitaxial films studied here were synthesized using an atomic layer-by-layer oxide molecular beam epitaxy (ALL-oxide-MBE) system. Single-crystal $\mathrm{LaSrAlO}_{4}$ (LSAO) and $\mathrm{SrTiO}_{3}$ (STO) substrates, $10 \times 10 \times 1 \mathrm{~mm}^{3}$ in size, epipolished with surface perpendicular to the [001] crystal axis were used. Further information about the MBE system and growing process has been published elsewhere [15]. The stoichiometry and doping level were controlled during the deposition by using well-calibrated MBE sources. The film growth and quality were monitored in real time using reflection high-energy electron diffraction (RHEED) system, and tested subsequently by atomic force microscopy (AFM) as well as by susceptibility and $\mathrm{x}$-ray diffraction measurements. Typical AFM figures are presented in Appendix B.

The $\mathrm{La}_{1.83} \mathrm{Sr}_{0.17} \mathrm{CuO}_{4}$ single crystal was grown by the traveling solvent floating zone technique. The sample originates from the same batch as the one analyzed in Ref. [16]. The asgrown cylindrical sample was cut in three slices of dimensions $\approx 1 \times 4 \times 20 \mathrm{~mm}^{3}$ with the $c$ axis normal to the larger plane. After cutting, the surface was cleaned by chemical etching in a $1 \%$ bromine/methanol solution, in order to restore the superconducting properties at the cut surfaces [17].

In order to study the magnetic field distribution in the superconducting state, $\mu \mathrm{SR}$ experiments were performed at the $\mu \mathrm{E} 4$ beamline [18] at the Paul Scherrer Institute. In a $\mu \mathrm{SR}$ experiment, spin-polarized positively charged muons $\left(\mu^{+}\right)$are implanted one at a time into the sample. In the presence of a local magnetic field $B_{\mu}$ at the muon stopping site the muon spin precesses at its Larmor frequency $\omega_{\mu}=\gamma_{\mu} B_{\mu}$, where $\gamma_{\mu}=8.51616 \times 10^{8} \operatorname{rad}(\mathrm{sT})^{-1}$ is the gyromagnetic ratio of the muon. The muon decays with a lifetime of $\tau_{\mu} \simeq 2.2 \mu \mathrm{s}$ into a positron and two neutrinos. Due to parity violation, the emission probability for the positron along the muon spin direction is enhanced. Each decay event is recorded together with the time difference $(t)$ between the implantation of the muon in the sample and its decay. The first detected by a muon detector placed on the muon trajectory before implantation, and the second via the decay positron. The positron count rate $N(t)$ allows one to determine the temporal evolution of the muon spin polarization $P(t)$ (time ensemble average):

$$
N(t)=N_{0} e^{-t / \tau_{\mu}}[1+A P(t)]+N_{\mathrm{bkg}} .
$$

Here, $N_{0}$ gives the scale of the counted positrons, $N_{\mathrm{bkg}}$ is a time-independent background of uncorrelated events, and $A$ is the observable decay asymmetry. The latter is a function of the positron energy and the solid angle of the positron detectors. The exponential function describes the radioactive muon decay. From the measured depolarization function $P(t)$ one can extract the local magnetic fields, field distributions, and field fluctuations present in the sample [19]. In bulk $\mu$ SR experiments, positively charged muons with an energy of $\approx 4.1 \mathrm{MeV}$ are used. They originate from the decay of a pion at rest, at the surface of the muon production target ("surface muons"). In this case, the mean stopping depth in condensed matter is of the order of $\sim 100 \mu \mathrm{m}$. To investigate thin films, low-energy $\mu \mathrm{SR}$ (LE- $\mu \mathrm{SR}$ ) makes use of epithermal muons $(\sim 15 \mathrm{eV})$. They are created by moderating surface muons $[12,20]$. After reacceleration, the final muon implantation energy is controlled by biasing the sample. Tuning the kinetic energy between 4.3 and $24.3 \mathrm{keV}$, mean implantation depths between 20 and $120 \mathrm{~nm}$ can be chosen. The in situ growth of a solid nitrogen film on top of the LSCO film allows to implant muons even closer to the film surface and study the LSCO-vacuum $/ \mathrm{N}_{2}$ interface.

We investigated four thin-film mosaic samples, each consisting of three or four $\mathrm{La}_{1.84} \mathrm{Sr}_{0.16} \mathrm{CuO}_{4}$ films with lateral dimensions $1 \times 1 \mathrm{~cm}^{2}$, and three different thicknesses $(\simeq 30 \mathrm{~nm}$, $\simeq 40 \mathrm{~nm}, \simeq 80 \mathrm{~nm}$ ), grown on two different substrates (LSAO and STO), as well as a mosaic of six $\mathrm{La}_{1.83} \mathrm{Sr}_{0.17} \mathrm{CuO}_{4}$ singlecrystal pieces, with total lateral dimensions of $\approx 1.5 \times 2 \mathrm{~cm}^{2}$. The samples were glued onto a nickel-coated aluminium plate using silver paint. In the analysis of the $\mu$ SR spectra, we have to take into account the contribution of muons stopping in the coating of the sample plate. $\mu^{+}$stopping in the sputtered ferromagnetic nickel layer of the sample plate quickly depolarizes. This contribution has the form

$$
P_{\mathrm{Ni}}(t)=2 / 3 \exp \left(-\Lambda_{\mathrm{Ni}} t\right)+1 / 3,
$$

with a depolarization rate of $\Lambda_{\mathrm{Ni}} \approx 40 \mu \mathrm{s}^{-1}$ [21]. One can exclude this nickel background contribution from the analysis by fitting the data in the time window $0.1 \mu \mathrm{s}<t<10 \mu \mathrm{s}$. All presented $\mu$ SR data were analyzed using MUSRFIT [22]. We performed temperature-dependent zero magnetic field (ZF) $\mu$ SR experiments to investigate possible magnetic orders in the sample and transverse magnetic field (TF) $\mu$ SR experiments to investigate the field distribution due to the vortex lattice. Using a cold-finger cryostat, temperatures down to $5 \mathrm{~K}$ were 
realized. The experiments were performed in ultrahigh vacuum at a pressure of about $10^{-9} \mathrm{mbar}$. The surface of the sample was perpendicular to the crystallographic axis [001], the transverse magnetic field was applied along the [001] direction, and the initial muon spin polarization was fixed at a certain angle in the plane of the sample. In this configuration, only the in-plane magnetic penetration depth is probed $\left(\lambda=\lambda_{a b}\right)$.

\section{EXPERIMENTAL RESULTS}

\section{A. Biaxial strain characterization}

In order to obtain biaxial strain, $\mathrm{La}_{1-x} \mathrm{Sr}_{x} \mathrm{CuO}_{4+\epsilon}$ thin films can be deposited on either a $\mathrm{LaSrAlO}_{4}(\mathrm{LSAO})$ or a $\mathrm{SrTiO}_{3}$ (STO) single-crystal substrate. The crystallographic $a$ axis of tetragonal LSAO is $\approx 0.6 \%$ smaller compared to LSCO, while the crystallographic $a$ axis of cubic STO is larger $(\approx 3.4 \%)$. A LSCO layer thinner than a critical thickness $h_{c}$ will accommodate for the mismatch and experience a biaxial strain. The strain relaxes through dislocations in thicker LSCO layer. For LSCO films grown on a STO substrate, a value of $h_{c} \simeq 18$ unit cells $(\approx 23 \mathrm{~nm})$ was obtained experimentally [23]. A theoretical model [24,25], taking into account the lattice mismatch and a LSCO Poisson ratio of $v \approx 0.3$ [26], gives $h_{c} \approx 15-25 \mathrm{~nm}$ in case of a STO substrate and $h_{c} \approx 75-100 \mathrm{~nm}$ for the LSAO substrate. For underdoped systems $(x \lesssim 0.15)$, the variation of $T_{c}$ with biaxial strain was associated with a modification of the oxygen content $(\epsilon)$ with the strain [27]. However, substantial variations of $T_{c}$ are still present for higher doping. In the overdoped regime $x \gtrsim 0.15$, the oxygen concentration is constant $(\epsilon=0)$ [2] and the value of $T_{c}$ still varies with biaxial strain. In order to neglect oxygen concentration effects, all the samples presented in this study have a strontium doping close to the optimal value of $x=0.16$. A measurement of the actual strain in each sample was performed using x-ray diffraction (Fig. 1). In reciprocal space, one compares the position of the Bragg diffraction spots of the substrate and sample. The Bragg peak of coordinates $\left(\begin{array}{lll}0 & 1 & 11\end{array}\right)$, in reciprocal space units $(\mathrm{H}, \mathrm{K}, \mathrm{L})$, is used for the thin films and compared to the Bragg peaks ( 0 $\left.\begin{array}{ll}1 & 11\end{array}\right)$ and $\left(\begin{array}{lll}0 & 1 & 3\end{array}\right)$ for the two different substrates LSAO and STO, respectively. Variations in the Bragg peak position in $\mathrm{L}$ are due to the different crystallographic $c$ axes, while in $\mathrm{K}$ it indicates the match or mismatch between in-plane lattice parameters, or the presence of a biaxial strain in the thin films. We quantified the amount of strain by comparing the length of the crystallographic $b$ axis of the unit cell for LSCO in thin film $\left(b_{\text {film }}\right)$, in the bulk phase $\left(b_{\text {bulk }}\right)$, and in the corresponding substrate $\left(b_{\text {substrate }}=b_{\mathrm{STO}}\right.$ or $\left.b_{\text {substrate }}=b_{\mathrm{LSAO}}\right)$ :

$$
\text { Strain }=1-\frac{b_{\text {film }}-b_{\text {substrate }}}{b_{\text {bulk }}-b_{\text {substrate }}},
$$

where $b_{\text {bulk }}=3.80515 \AA, b_{\mathrm{STO}}=3.905 \AA, b_{\mathrm{LSAO}}=3.75 \AA$ and for the film

$$
b_{\text {film }}=\frac{b_{\text {substrate }}}{K(\text { LSCO })-K(\text { substrate })+1} .
$$

Here, $K$ (LSCO) and $K$ (substrate) are the positions of the Bragg peaks for the film and substrate determined on Fig. 1. As expected, a thickness of $40 \mathrm{~nm}$ is above $h_{c}$ for a LSCO film grown on STO and thus it is fully relaxed, while the same thickness or thinner on LSAO is clearly less than $h_{c}$ and the
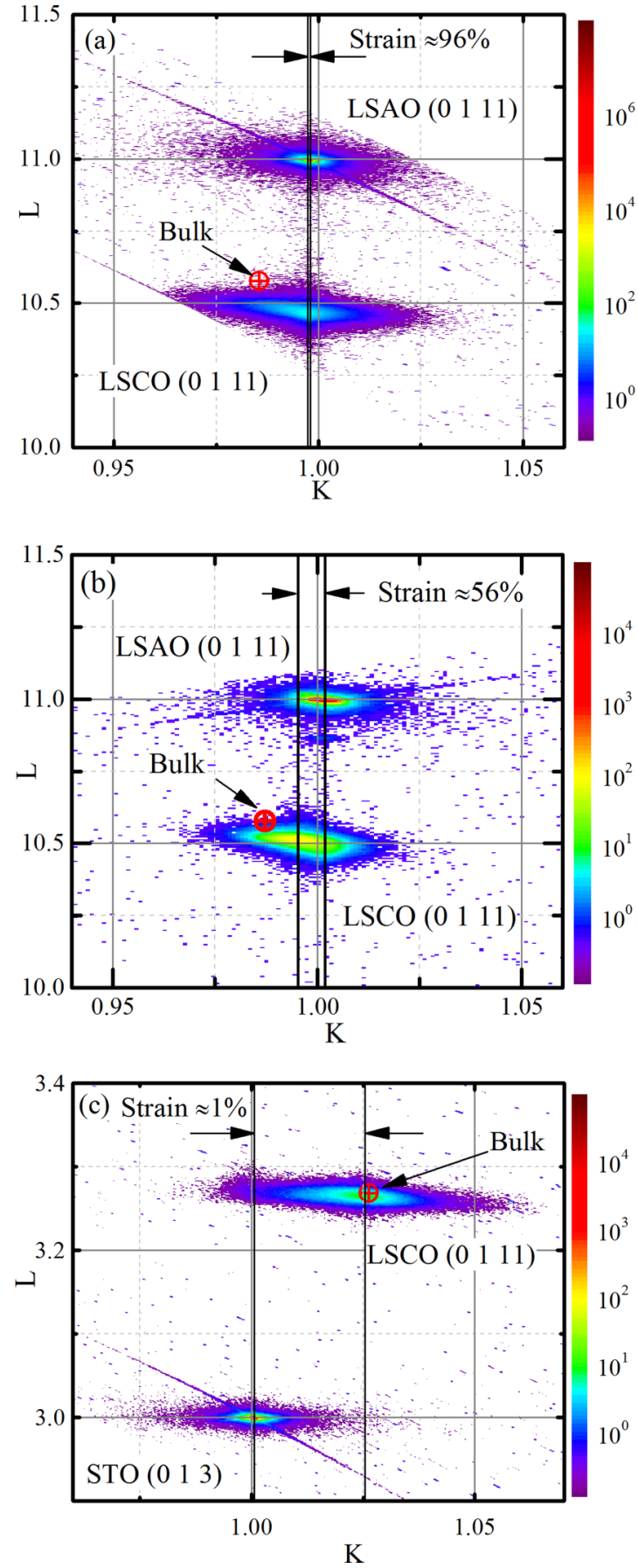

FIG. 1. Typical x-ray diffraction reciprocal space images for LSCO films grown on LSAO and STO substrates. The dimensionless coordinates are relative to the reciprocal crystallographic axes of the substrates. The 40-nm-thick LSCO film grown on a LSAO substrate is under compressive strain (a) while no strain remains on the 40-nm LSCO film grown on a STO substrate (c). For the 80-nm-thick LSCO film grown on a LSAO substrate, an intermediate strain state is observed (b).

resulting films are fully strained. An 80-nm-thick film grown on LSAO is close to the critical thickness and we obtained a rare intermediate strain. Note that strain release is expected to occur via dislocations at the interface [28], therefore, the strain is expected to be uniform through the full LSCO film thickness. 


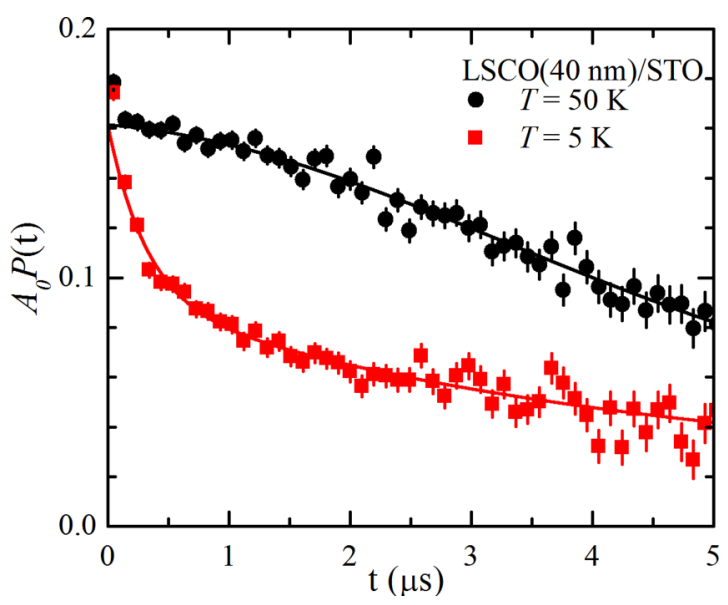

FIG. 2. $\mu$ SR asymmetry in zero external magnetic field for a 40-nm thin film of LSCO grown on a STO substrate. The implantation energy of the muons was $E_{k}=4.3 \mathrm{keV}$. At $50 \mathrm{~K}$ the asymmetry can be fitted with Eq. (6), while at $5 \mathrm{~K}$ an additional exponential decay must be introduced. The first point for $t<0.1 \mu \mathrm{s}$ is not taken into account for the fit, as it contains a contribution from the muons stopping in the nickel sample holder.

The narrow width of the LSCO Bragg peaks strengthens this assumption.

\section{B. Zero-magnetic-field $\mu \mathrm{SR}$ measurements}

The zero-field (ZF) $\mu$ SR polarization of a paramagnetic system should be dominated by the nuclear spins only. Under these conditions, the time spectra take the form of the so-called Gauss Kubo-Toyabe function [19]

$$
A_{0} P_{\mathrm{ZF}}(t)=A_{0}\left\{\frac{1}{3}+\frac{2}{3}\left[1-(\sigma t)^{2}\right] \exp \left[-1 / 2(\sigma t)^{2}\right]\right\},
$$

where $A_{0}$ is the instrumental asymmetry, and $\sigma=\gamma_{\mu} \Delta_{\mathrm{G}} \cdot \Delta_{\mathrm{G}}$ is the root mean square of the nuclear dipole field distribution at the muon site. $A(t)=A_{0} P_{\mathrm{ZF}}(t)$ is called the asymmetry. We performed ZF $\mu$ SR experiments adapting the muon implantation energy such that the mean implantation depth is roughly in the center of the film and that the full implantation profile lies within the film limits. For the 40-nm-thick film we used an implantation energy of $4.3 \mathrm{keV}$ corresponding to a mean implantation depth of $\sim 20 \mathrm{~nm}$. For the single crystal, the measurement was performed at the surface $(4.3 \mathrm{keV})$ and deeper in the bulk $(24.3 \mathrm{keV})$. In the single crystal and for all the different thin films grown on LSAO substrates, the asymmetry is following Eq. (6) as expected from nuclear dipole broadening.

However, for LSCO thin films grown on STO, the polarization as given by Eq. (6) is only describing the data at high temperatures. At low temperatures, the asymmetry shows a very strong initial depolarization (see Fig. 2). This is a typical signature of a magnetic state. In fact, the LSCO phase diagram shows at low temperatures in the underdoped region a magnetic ground state mostly called a cluster spin-glass phase [29]. This is also found in LSCO thin films grown on LSAO [30], comparable in thickness to the films presented here. However, the LSCO films studied here are all optimally doped. At optimal doping, no magnetic signature was previously reported, neither in the bulk nor in LSCO thin films.

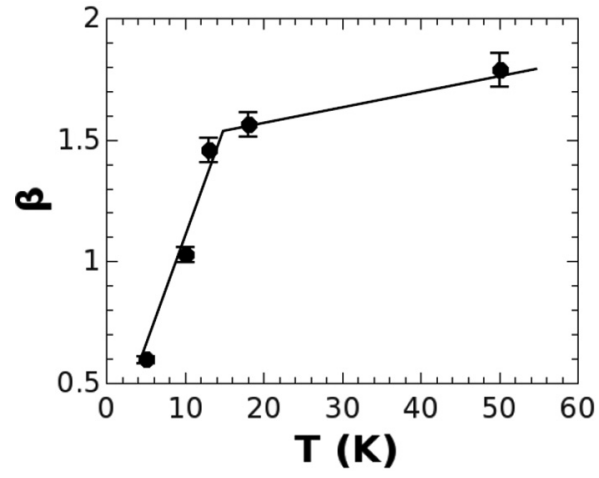

FIG. 3. Temperature dependence of the parameter $\beta$ of Eq. (7), fitting the $\mu$ SR asymmetry in zero external magnetic field for a $40-\mathrm{nm}$ thin film of LSCO grown on a STO substrate. The presence of a low-temperature magnetic phase is revealed.

In order to describe the ZF asymmetry for different implantation energies and in the whole temperature range we used the following ansatz:

$$
A_{0} P_{\mathrm{ZF}}(t)=A_{0} \exp \left[-(\lambda t)^{\beta}\right] .
$$

Interestingly, the temperature dependence of $\beta$ (Fig. 3) obtained from data in the center of the LSCO film [muon implantation simulated in Fig. 4(b), $E=4.3 \mathrm{keV}$ ] shows a strong change at $T_{c}=14 \mathrm{~K}$ (see Table I for the different $T_{c}$ values), indicating that the apparition of the magnetic ground state coincides with the superconducting transition. The square-root time dependence of $P_{Z} F(t)$ observed at low temperatures $(\beta \simeq 1 / 2$ ) was previously observed by Uemura in the context of canonical spin glasses for temperatures slightly above the glass transition temperature $T_{g}$ [31]. On the other hand, for a dense randomly oriented, static magnetic state, one can show that $\beta=2$ [19].

The depth dependence of the magnetic state in the 40-nm-thick LSCO film grown on STO was studied using different muon implantation energies (see Fig. 4). In order to cover a large implantation depth $\langle z\rangle$ range, two sets of experiments where carried out: (i) the $\mu^{+}$were implanted as depicted in Fig. 4(b), and (ii) an 80-nm-thick $\mathrm{N}_{2}$ overlayer was grown in situ, resulting in $\mu^{+}$stopping profiles as shown in Fig. 4(a). The implantation profiles were simulated using the TRIM.SP [32] Monte Carlo code. The fraction of muons stopping in the LSCO film $\left(f_{S}\right)$ was extracted from these simulations. Both sets of measurements contain a background contribution of the STO substrate and the $\mathrm{N}_{2}$ overlayer, respectively. The background contribution was subtracted from the asymmetry time spectra using

$$
A_{S}(t)=\frac{A(t)-\left(1-f_{S}\right) A_{\mathrm{bkg}}(t)}{f_{S}} .
$$

The resulting asymmetries $A_{S}(t)$ are shown in Fig. 4(c) and were fitted using Eq. (7).

Figures 4(d) and 4(e) show the depolarization rate $\lambda$ and the exponent $\beta$ as a function of mean implantation depth $\langle z\rangle$, respectively. The depolarization rate $\lambda$ is decreasing towards the STO interface. Larger values of $\lambda$ correspond to a larger effective "quasistatic" magnetic moment on the muon time scale. Together with the depth dependence of $\beta$, this suggests 


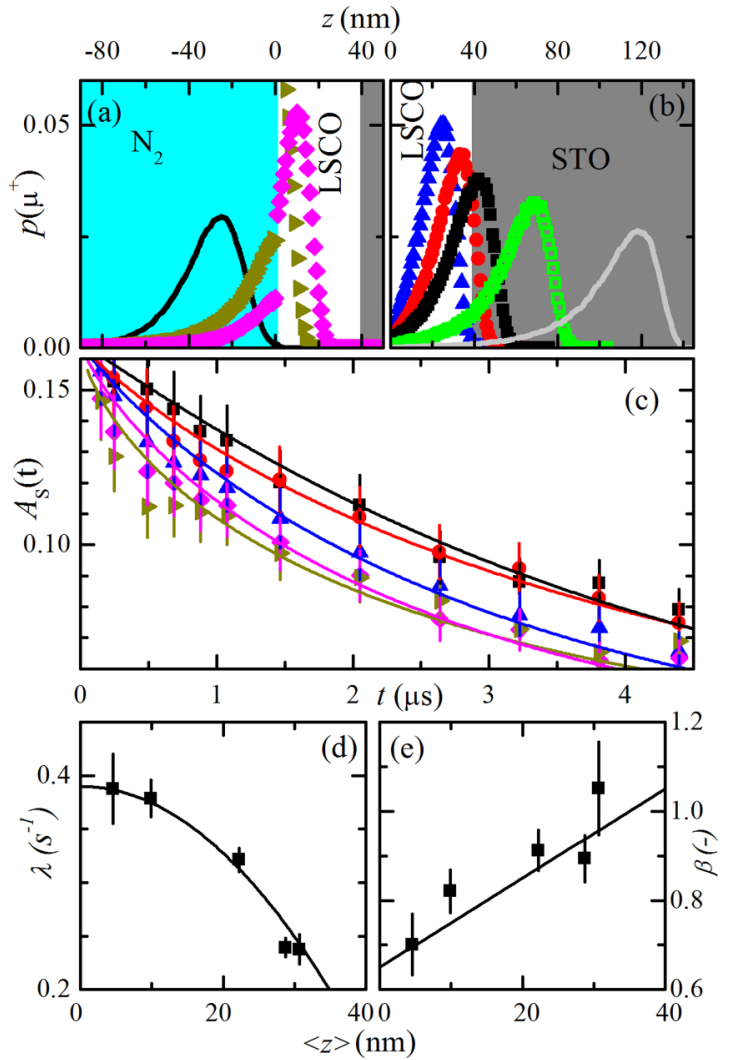

FIG. 4. Muon implantation depth probabilities $p\left(\mu^{+}\right)$for the 40-nm thick LSCO film on STO, (a) with a 80 -nm solid- $\mathrm{N}_{2}$ overlayer ( $E_{\mu}=4.3 \mathrm{keV}$ solid black line, $7.3 \mathrm{keV}$ green side triangles, $9.3 \mathrm{keV}$ pink diamonds), and (b) without solid- $\mathrm{N}_{2}$ overlayer $\left(E_{\mu}=4: 3 \mathrm{keV}\right.$ blue triangles, $6.3 \mathrm{keV}$ red circles, $8.3 \mathrm{keV}$ black squares, $14.3 \mathrm{keV}$ green squares, $24.3 \mathrm{keV}$ solid gray line). The solid lines correspond to the implantation depth probabilities used to calculate the two background muon depolarization signal. (c) ZF asymmetry $A_{S}$ from the LSCO layer at $5 \mathrm{~K}$ as defined by Eq. (8). Solid lines are fits of Eq. (7) to $A_{S}$. The color of the fit lines as well as the color and shape of the data points match the ones of the implantation depth probability from the corresponding muons in panels (a) and (b).

that the magnetic state is dynamic, and is becoming more static on approaching the LSCO surface. This suggests that the surface of the LSCO film is pinning the magnetic state.

This behavior could originate from a motional narrowing effect which, if correct, would mean that the magnetic state in the center of the film and at the LSCO/STO interface is dynamic, but pinned towards the surface where the magnetic state is becoming "quasistatic." Note that the surfaces of the LSCO films grown on STO substrates are atomically smooth (see AFM images in Appendix B).

\section{Transverse field $\mu \mathrm{SR}$ measurements}

For a superconductor in the vortex state, the vortex lattice generates a magnetic field distribution $p_{\mathrm{VL}}(B)$ in the sample. The field distribution due to the vortex lattice is asymmetric, characterized by a variance $\left(\sigma_{\mathrm{VL}}^{2}\right)$ and a positive skewness $\left[\theta=M_{3}^{1 / 3} / M_{2}^{1 / 2}\right.$, with $M_{n}$ the $n$th moment of $\left.p_{\mathrm{VL}}(B)\right]$, that are uniquely determined by the values of the magnetic penetration
TABLE I. Summary of the single-crystal and thin-film results. From the magnetic field dependence of $\sigma_{\mathrm{VL}}\left(B_{\mu}\right)$ obtained in the $\mu$ SR experiment, the magnetic penetration depth is calculated using the numerical London model, without dead layer $\lambda(\delta=0)$ and with a dead layer $\lambda(\delta>0)$. In the single crystal, a dead layer of $\delta=$ $50 \mathrm{~nm}$ was assumed, while $\delta=7.5 \mathrm{~nm}$ was used for the thin films, justified by the film surface roughness observed in the AFM images (see Appendix C).

\begin{tabular}{lcccc}
\hline \hline Sample & $T_{c}(\mathrm{~K})$ & $d(\mathrm{~nm})$ & $\lambda(\delta=0)$ & $\lambda(\delta>0)$ \\
\hline Bulk & $34.4(5)$ & $\approx 10^{5}$ & $270 / 200$ & $270 / 200$ \\
Surface & $34.4(5)$ & $\approx 200$ & 285 & 275 \\
LSCO $(80 \mathrm{~nm}) / \mathrm{LSAO}$ & $32(1)$ & $80(2)$ & 385 & 375 \\
LSCO $(42 \mathrm{~nm}) / \mathrm{LSAO}$ & $32(1)$ & $42(2)$ & 330 & 275 \\
LSCO $(30 \mathrm{~nm}) / \mathrm{LSAO}$ & $33(1)$ & $30(2)$ & 375 & 280 \\
LSCO $(39 \mathrm{~nm}) /$ STO & $14(1)$ & $39(2)$ & & \\
\hline \hline
\end{tabular}

depth $\lambda$ and the coherence length $\xi$ of the superconducting material. For large values of $\lambda$, such as in thin films due to continuity at the interfaces, $\theta$ is small. In a LE- $\mu$ SR experiment, due to the limited event rate, the statistics does not allow to resolve $\theta$. For this reason, in this work, we assume a Gaussian field distribution $(\theta=0)$ with variance $\sigma_{\mathrm{VL}}^{2}$. The magnetic field dependence of $\sigma_{\mathrm{VL}}$ in bulk material can be calculated theoretically following the work of Brandt [33] (inset of Fig. 5).

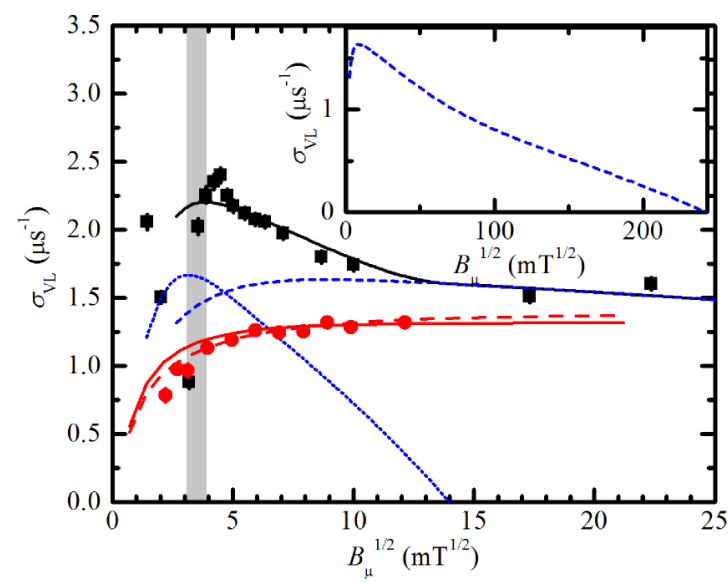

FIG. 5. The magnetic field dependence of the vortex lattice depolarization rate $\sigma_{\mathrm{VL}}$, as defined in the text, is plotted for the LSCO single crystal. The implantation energies were $E \sim 4 \mathrm{MeV}$ (black squares) and $E=21 \mathrm{keV}$ (red circles) corresponding to mean implantation depths of about $100 \mu \mathrm{m}$ and $100 \mathrm{~nm}$, respectively. The dashed and dotted blue lines represent the calculated field distribution for two different values of $\lambda(270$ and $200 \mathrm{~nm})$, and to different upper critical fields of 60 and $0.2 \mathrm{~T}$, respectively, calculated following the Brandt numerical model [33]. The solid black line is the convolution of the two contributions. The red solid and dashed lines are calculations using the numerical London model for a crystal with fully superconducting surface $(\lambda=285 \mathrm{~nm})$ and with a "dead layer" of $50 \mathrm{~nm}(\lambda=275 \mathrm{~nm})$, respectively. The gray region represents the lower critical field $H_{c 1}$ for parameters $\mu_{0} H_{c 2}=60 \mathrm{~T}$ and $\lambda=220-290 \mathrm{~nm}$. 
In a transverse-field muon spin rotation experiment (TF- $\mu \mathrm{SR}$ ), the magnetic field is applied perpendicularly to the muon spin orientation, and the polarization function $P_{\mathrm{TF}}(t)$ can often be described by

$$
P_{\mathrm{TF}}(t)=\cos \left(2 \pi \times v_{\mu} t+\phi\right) \exp \left(-\sigma^{2} t^{2} / 2\right) .
$$

Here, $\phi$ is the initial phase of the muon spin with respect to the positron detector. The field distribution $p(B)$ of Gaussian width $\sigma_{\mathrm{N}}$ in the normal state is due to the contribution of the nuclear magnetic moments. In the superconducting state $\sigma$ is given by the convolution of the second moment of the $z$-component field distribution generated by the magnetic induction in the vortex lattice $\sigma_{\mathrm{VL}}$ and the nuclear moment contributions in the superconducting state:

$$
\sigma^{2}=\sigma_{\mathrm{VL}}^{2}+\sigma_{\mathrm{N}}^{2}
$$

The value of $\sigma_{\mathrm{VL}}$ was extracted for different applied external magnetic fields $\mu_{0} H$, recording a $\mu$ SR spectra in the normal $(T=50 \mathrm{~K})$ and, after field cooling, in the superconducting $(T=5 \mathrm{~K})$ states (see Appendix $\mathrm{C}$ for representative examples):

$$
\sigma_{\mathrm{VL}}(H)=\sqrt{\sigma(5 \mathrm{~K}, H)^{2}-\sigma(50 \mathrm{~K}, H)^{2}} .
$$

Generally, in the bulk of superconducting samples, $\sigma_{\mathrm{VL}}(H)$ increases rapidly with increasing $H$ and reaches a maximum at a field $H_{m}$ corresponding to a distance between the vortices of the order of the magnetic penetration depth $\lambda$ [34]. In superconductors with large Ginzburg-Landau parameter, the maximum of $\sigma_{\mathrm{VL}}(H)$ is expected to be broad and in the limit $H / H_{c 2} \ll 1$ and $H>H_{c 1}, \sigma_{\mathrm{VL}}$ is usually assumed to be only weakly field dependent [19]. Here, $H_{c 1}$ is the lower superconducting critical field. In this limit and for an hexagonal vortex lattice, the second moment of the field distribution is given by

$$
\sigma_{\mathrm{VL}}\left(H_{m}\right)=0.06092 \times \Phi_{0} / \lambda^{2},
$$

where $\Phi_{0}$ is the flux quantum.

In thin films, the size of the vortex is extended at the interfaces for continuity reasons and an effective magnetic penetration depth $\left(\lambda_{\text {eff }}\right)$ must be considered. The effective value can be related to the intrinsic magnetic penetration depth $\lambda$ via the relation [35]

$$
\lambda_{\mathrm{eff}}=\lambda \operatorname{coth}(\lambda / d) .
$$

In the limit $d \ll \lambda$, this relation can be written as

$$
\lambda_{\text {eff }} \simeq \lambda^{2} / d .
$$

The effective magnetic penetration depth gives the right order of magnitude of the reduction of $\sigma_{\mathrm{VL}}\left(H_{m}\right)$ in thin films compared to bulk samples, however, the full field dependence of $\sigma_{\mathrm{VL}}(H)$ is modified in thin films compared to the bulk. The low-field $\left(H \ll H_{c 2}\right)$ dependence of the second moment of the $z$ component of the magnetic induction in the vortex state of a strong type-II superconductor can be obtained within the London framework, for any $z$ position, as detailed in the Appendix A. In the following, the experimental results are compared either to the bulk numerical model of Brandt [33] or to the thin-film numerical London model.

\section{Single crystal}

The field dependence of $\sigma_{\mathrm{VL}}\left(B_{\mu}\right)$ for an optimally doped $\mathrm{La}_{1.83} \mathrm{Sr}_{0.17} \mathrm{CuO}_{4}$ single crystal is presented in Fig. 5. The black squares represent the bulk data of the single crystal since the mean implantation depth was about $\approx 100 \mu \mathrm{m}$, using surface muons. The open red circles instead represent data measured close to the surface at a depth of about $100 \mathrm{~nm}$ using low-energy muons with a kinetic energy of $21 \mathrm{keV}$. The upper critical field for optimally doped LSCO was reported to be $\mu_{0} H_{c 2} \approx$ $60 \mathrm{~T}$ [36]. For the bulk, the field dependence of $\sigma_{\mathrm{VL}}$ can be calculated following Ref. [33] (inset of Fig. 5). In the field range investigated, this model predicts an approximately constant value (dashed line in Fig. 5) of $\sigma_{\mathrm{VL}}$ in disagreement with our experimental results.

A better description of the bulk data is achieved by the convolution of two different contributions to the field distribution $\sigma_{\mathrm{VL}}$. This is justified by the proposal that $\mathrm{La}_{1.84} \mathrm{Sr}_{0.16} \mathrm{CuO}_{4}$ is a multigap system [16]. The two different electronic bands $(i)$ causing the multigap superconductivity would have their own superconducting parameters

$$
\lambda_{i}=\sqrt{m_{i}^{\star} c^{2} /\left(4 \pi n_{i} e\right)} \text { and } \xi_{i}=\hbar v_{\mathrm{F} i} /\left(\pi \Delta_{i}\right),
$$

leading to two different field distributions. Here, $e$ is the electron charge, $m_{i}^{\star}, n_{i}, v_{\mathrm{F} i}$ and $\Delta_{i}$ are the band-specific effective mass, number density, Fermi velocity, and superconducting gap. Using the literature values for the upper critical field for the two bands ( 60 and $0.2 \mathrm{~T}$ ) [16,36] and adjusting the values of the magnetic penetration depth $\lambda$, a qualitative agreement (full line in Fig. 5) is obtained for the bulk data. However, the shape and position of the maximum in $\sigma_{\mathrm{VL}}\left(B_{\mu}\right)$ cannot be reproduced exactly. Below the first critical field $\left(H_{c 1}\right)$, the theory predicts that the sample is in the Meissner state at base temperature $(5 \mathrm{~K})$. However, as this state is reached by cooling the sample under magnetic field (field cooling), the vortex state is realized during the cooling procedure. Since bulk LSCO samples are known to exhibit strong pinning centers, a disordered vortex network is expected at $5 \mathrm{~K}$, explaining the difference between the model (black line in Fig. 5) and the experimental data (black squares in Fig. 5) below $H_{c 1}$.

At the surface of the single crystals, the diameter of the vortices increases, leading to a larger value of the effective magnetic penetration depth $\left(\lambda_{\text {eff }}\right)$. As a consequence, the value of $\sigma_{\mathrm{VL}} \propto \lambda_{\text {eff }}^{-2}$ decreases. Using the numerical London model, we found that the initial slope is strongly dependent on the proximity to the interface. When the crystal is assumed to be fully superconducting (full red line in Fig. 5), a substantial deviation is observed between the model and the experimental results. The simulation becomes much better by introducing a nonsuperconducting surface layer of $\sim 50 \mathrm{~nm}$ (dashed red line in Fig. 5). LE- $\mu$ SR measurements in the Meissner state also found such a "dead layer" $\delta$ [37]. In those measurements, the samples are zero field cooled and at lowest temperature an external field $H<H_{c 1}$ is applied. This allows to measure directly the screened magnetic field profile at the surface. Theoretical calculations [38] show that surface roughness is leading to an effective "dead layer" of a couple of nanometers due to a curvature variation of the magnetic field at the surface. Unfortunately, a similar quantitative calculation for the vortex state is missing. The sawing process and chemical 


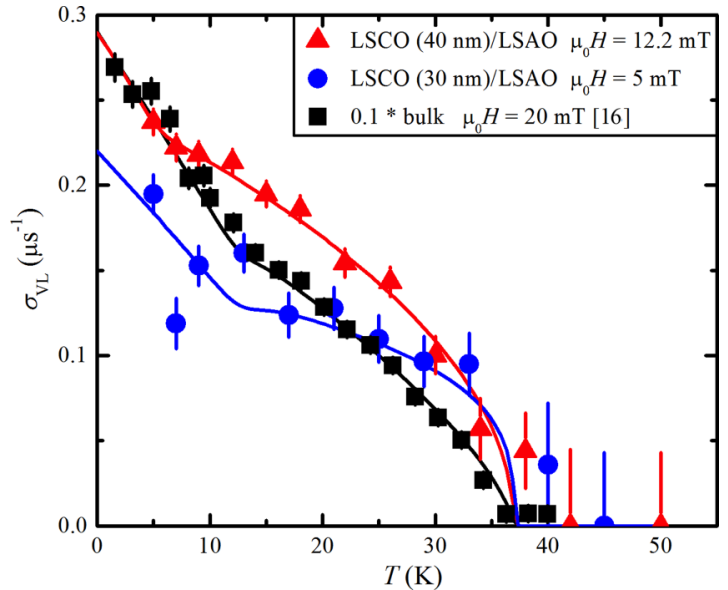

FIG. 6. Temperature dependence of $\sigma_{\mathrm{VL}}$ for a 30-nm (blue circles) and a 40-nm (red triangles) thin film of LSCO grown on LSAO substrates. For comparison, the bulk values from Ref. [16] divided by a factor of 10 are shown (black squares). The solid lines are guides to the eyes.

treatment could certainly explain the large- $\delta$ value observed in the mosaic sample of the single crystal as the surface is certainly disordered due to surface roughness and chemical modification such as a modification of oxygen concentration and local strain.

\section{Magnetic penetration depth in LSCO films grown on LSAO substrates}

The temperature evolution of $\sigma_{\mathrm{VL}}(T)$ for two thin films of LSCO grown on LSAO substrates is presented in Fig. 6, compared to the bulk data of Ref. [16]. The temperature dependence of $\sigma_{\mathrm{VL}}(T)$ is very similar in the films compared to the bulk. The reduction of a factor $\sim 10$ in $\sigma_{\mathrm{VL}}$ between the films and the single crystal is partially explained by the increase of the effective magnetic penetration depth in the films. For a 40-nm-thick film with a magnetic penetration depth $\lambda>200 \mathrm{~nm}$, one would expect from Eqs. (12) and (14) a reduction of $\sigma_{\mathrm{VL}}$, in the film compared to the bulk by a factor $\sigma_{V L}($ Bulk $) / \sigma_{V L}($ Film $) \simeq(\lambda / d)^{2}>25$, much more than experimentally observed. This indicates that a specific model, such as the numerical London model, must be applied to better describe quantitatively $\sigma_{\mathrm{VL}}\left(B_{m}\right)$ in thin films. The relation between $\sigma_{\mathrm{VL}}$ and the second moment of the magnetic field distribution due to the vortex lattice is outlined in the Appendix A.

The field dependence of the second moment of the field distribution probed in TF $\mu$ SR in a $40-\mathrm{nm}$ film is plotted in Fig. 7. Experimentally, $\sigma_{\mathrm{VL}}$ is found to increase practically as the square root of the applied field, a behavior very difficult to catch with the simulation presented here. Indeed, reducing the superconducting thickness with the introduction of nonsuperconducting layers at the interfaces leads to a better agreement. The AFM images (see Appendix B) show that LSCO thin films under compressive strain have a particular growth mode, with a columnar structure on the first $\sim 7 \mathrm{~nm}$. Such a structure might be detrimental to superconductivity and justifies a large nonsuperconducting layer. However, much larger nonsuperconducting layers would lead to a very small magnetic penetration depth which is unexpected as cuprates

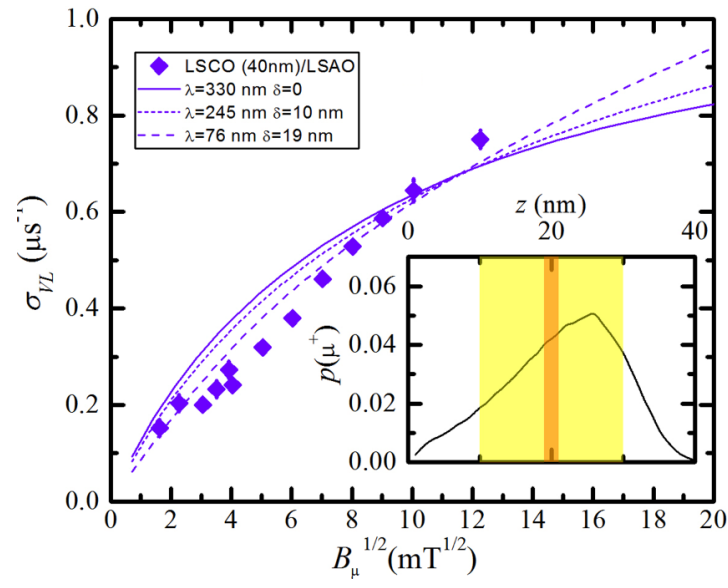

FIG. 7. Field evolution of $\sigma_{\mathrm{VL}}$ for a compressively strained 40-nm-thick LSCO thin film on LSAO. The lines depict simulations within the numerical London model, considering different superconducting thicknesses, i.e. with or without nonsuperconducting layers $\delta$ at the interfaces. The magnetic penetration depth is adjusted for each thickness to obtain the best fit. The implantation depth probability distribution is plotted as inset together with the superconducting thicknesses used for the different simulations.

have a low carrier density and an enhanced effective mass, which according to Eq. (1) leads to a large penetration depth. On the contrary, it is certainly more the simple London approach with a steplike variation of the carrier concentration at the interface and a deltalike core that is inappropriate and more theoretical research is needed to fully grasp the physics of these films. However, the simple London approach has the merit to give an estimate for the value of the fundamental property of the superconductor that is the intrinsic magnetic penetration depth.

The magnetic field dependence of $\sigma_{\mathrm{VL}}\left(B_{\mu}\right)$ for all the different films investigated in this study is shown in Fig. 8. The

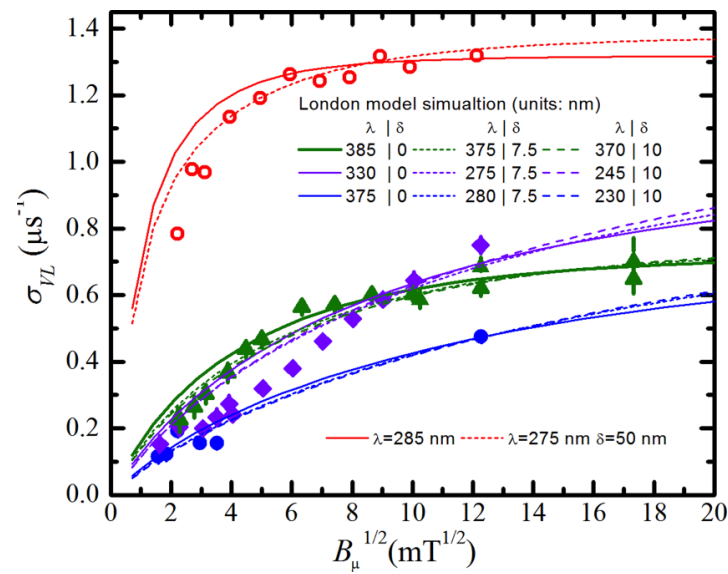

FIG. 8. Magnetic field dependence of $\sigma_{\mathrm{VL}}\left(B_{\mu}\right)$ for LSCO films of various thicknesses $d$, grown on LSAO: $d=30 \mathrm{~nm}$ (blue circles), $d=40 \mathrm{~nm}$ (violet diamonds), $d=80 \mathrm{~nm}$ (green triangles). In addition, $\sigma_{\mathrm{VL}}\left(B_{\mu}\right)$ measured at the surface of a single crystal is plotted as empty red circles. Full and dashed lines are the model described in the text for different values of magnetic penetration depth $\lambda$ and dead layer $\delta$. 
numerical London model shows that the initial slope is given by the superconducting film thickness and we observe indeed that $d \sigma_{\mathrm{VL}} / d B_{\mu}$ increases with the film thickness. At a given magnetic field, we expect the value of $\sigma_{\mathrm{VL}}\left(B_{\mu}\right)$ to increase with film thickness due to the decrease of the effective magnetic penetration depth $\lambda_{\text {eff }}$, if the intrinsic magnetic penetration depth $\lambda$ is not or only weakly affected. Unexpectedly, a crossover is observed between $\sigma_{\mathrm{VL}}\left(B_{\mu}\right)$ of the $80-\mathrm{nm}$ and 40 -nm thick film at $B_{\mu} \sim 70 \mathrm{mT}$. The crossover is already visible on the bare experimental results (see Appendix C). This indicates that the intrinsic magnetic penetration depth is smaller in the 40-nm-thick film than in the 80-nm one. There are two main differences between these films: the thickness and the biaxial strain. The 40-nm-thick film is fully compressively strained, while the $80-\mathrm{nm}$ one is partially relaxed. The effect of thickness is reflected in the initial slope, therefore, we conclude that the biaxial compressive strain reduces the value of the intrinsic magnetic penetration depth.

Using the numerical London model one can quantify the variation of intrinsic magnetic penetration depth assuming a fixed dead layer. For atomically smooth thin films we expect surface roughness and surface chemical alteration to lead to a small value of $\delta$. On the other hand, the 30- and 40-nm films have similar compressive strain and therefore their intrinsic magnetic penetration depth should match. This suggests that the dead layer of our atomically smooth films is in the range $7.5-10 \mathrm{~nm}$. Even if the model is not exact, such a large value clearly indicates that the superconductivity at the surface is drastically modified compared to the bulk. Using a dead layer of $\delta=7.5 \mathrm{~nm}$ the intrinsic magnetic penetration depths for the films compared to the bulk are presented in Table I.

\section{DISCUSSION}

\section{A. Magnetism}

The zero-field $\mu \mathrm{SR}$ experiments show the presence of "quasistatic" magnetism in optimally doped 40-nm thin LSCO films grown on STO. No signs of magnetism were found in optimally doped LSCO films grown on LSAO. The same is true for the near-surface measurements $(\sim 100 \mathrm{~nm})$ on optimally doped LSCO single crystals. The magnetic phase found in LSCO/STO resembles rather the phase of canonical spin glasses than the cluster spin-glass phase found in underdoped LSCO. This points to a dynamic short-range magnetic order. The studied LSCO/STO film has no biaxial strain, however, the mismatch of the film and substrate crystallographic axis will generate dislocations. We can reasonably assume that such defects help to slow down high-frequency magnetic fluctuations and hence stabilize a magnetic order.

A magnetic order in an applied magnetic field of $7.5 \mathrm{~T}$ appearing in the vortices of optimally doped LSCO was evidenced by neutron scattering experiments [39]. Note that such an applied magnetic field is much higher than the ones used in this study. As vortices extend toward the surfaces of a superconductor, such a magnetic order is also mainly present at the surface. Later, field-induced surface ferromagnetism was reported by $\mathrm{x}$-ray magnetic circular dichroism and was attributed to a canting of $\mathrm{Cu}^{2+}$ spins antiferromagnetically interacting through a distortion of the $\mathrm{CuO}_{6}$ octahedra (Dzyaloshinskii-Moriya interaction) [40].
The present experiment is fully compatible with the existing literature, however, we can be more precise as the observed magnetic order only appears "quasistatic" on the muon time scale, at the film surface, and for temperatures below the superconducting phase transition. This might be due to different structural or chemical properties of the surface compared to the bulk. It might also indicate competing order between magnetism and a superconducting phase weakened at the film surface.

\section{B. Superconductivity}

As discussed previously, the magnetic field dependence of the magnetic field distribution measured by $\mu$ SR in the bulk of the single crystal is best described using a multigap model of superconductivity. This is not surprising as multigap features seem to be a systematic characteristic of unconventional superconductors with numerous reports in the recent literature. Multigap superconductivity is found in heavy-fermion systems [41], uranium-based systems [42], pnictides [43-45], and cuprates $[16,46,47]$. Interestingly, the multigap feature disappears at the surface of the superconductor. This is consistent with the variation of electronic band structure suggested in the literature. Indeed, surface sensitive experiments find a single hole band (e.g. ARPES [48]), while multiple bands are found with bulk sensitive techniques such as the Nernst effect [49] (A hole and an electron bands) or de-Haas van Alphen quantum oscillations [50] (Two frequencies for two electronic bands with different effective masses). It remains a challenge to understand the full dependence of $\sigma_{\mathrm{VL}}\left(B_{\mu}\right)$ which we revealed to be more complex than expected by the calculation, within the London framework. A solution of the Ginzburg-Landau equations for superconducting films was treated by Brandt [51]. Unfortunately, a mistake must be present in the numerical formulation of the solution presented in this paper. Indeed, despite several implementations of the code, we could not reproduce the results of the paper.

Even though the numerical London model has its shortcomings, it allows to identify the following things: first, it is indicating the particularity of surfaces. Indeed, the need of a large "dead layer" leads us to reconsider the boundary conditions of the superconducting state and notably how the superconducting carrier concentration varies toward the interfaces. Second, it allowed us to estimate the evolution of the magnetic penetration depth with biaxial strain.

With the introduction of an appropriate "dead layer," we obtain that all the samples except the 80-nm LSCO on LSAO have an intrinsic magnetic penetration depth of $\lambda \sim 275 \mathrm{~nm}$. From the shape of the field dependence of $\sigma_{\mathrm{VL}}\left(B_{\mu}\right)$, we also concluded that biaxial strain reduces $\lambda$. We might understand the larger value of $\lambda$ for this particular film as follows: In thin films, reduced dimensionality is detrimental for superconductivity and leads to a general increase of the magnetic penetration depth, probably through a reduction of the number density $n_{S}$. Biaxial compressive strain, on the contrary, is favorable to superconductivity, as reported by several transport studies [5,9-11]. It is then a coincidence that the same value is obtained in compressively strained films and in the bulk which results to an almost identical $T_{c}$. The large value of $T_{c}$ observed in the 80-nm LSCO grown on LSAO can be due to some 
inhomogeneity, with a portion of the film fully compressed at the substrate interface giving the large- $T_{c}$ value, while the $\lambda$ value is mostly determined by the partially relaxed bulk portion of the film. Additional measurements are certainly required to get a full picture of the physics of this system as well as a better understanding of vortex lattice and superconducting properties in thin films.

\section{CONCLUSION}

We have studied the magnetic field distribution in the vortex state, at low temperatures in thin $\mathrm{La}_{1.84} \mathrm{Sr}_{0.16} \mathrm{CuO}_{4}$ superconducting films under biaxial strain and in a single crystal, as well as the magnetic behavior of the films at low temperatures in absence of an externally applied magnetic field. Bulk and surface superconductivity are found to be drastically different.

In zero-field $\mu \mathrm{SR}$ experiments on a fully relaxed film grown on $\mathrm{SrTiO}_{3}$ substrate, we have established that at the surface small disturbances (probably dislocations) can lead to the presence of a "quasistatic" magnetic phase. In transverse-field $\mu \mathrm{SR}$ experiments on thin films, the dependence of the second moment $\sigma_{\mathrm{VL}}$ of the measured field distribution, caused by the vortex lattice, does not fully follow the theoretical predictions derived from a London model. In the bulk, the field dependence $\sigma_{\mathrm{VL}}\left(B_{\mu}\right)$ can be quantitatively understood assuming multigap superconductivity. However, the multigap features disappear at the surface. This result indicates a drastic modification of the electronic band structure. Starting with the often reported single band structure at the surface ( $\sim 100$ first nanometres) the electronic structure evolves to multiband in the bulk. Furthermore, one must add a substantial nonsuperconducting "dead layer" in order to reproduce the experimental variation of $\sigma_{V L}\left(B_{\mu}\right)$. This indicates that the surface is either not or less (smaller number density) superconducting than the bulk, on a length scale of $\sim 7.5 \mathrm{~nm}$, that is about three times the coherence length. Two results that force us to reconsider the boundary conditions of the superconducting state. A prototypical question is how does the number density $n_{S}$ vary at the interfaces? It also clearly indicates nonequivalent surface and bulk superconductivity.

Finally, our results suggest that the magnetic penetration depth is decreased by the application of a biaxial compressive strain. If one considers that compressive strain is in general favorable to superconductivity, this indicates, following Eq. (1), that biaxial strain influences the number density of the superconducting carriers and not its pairing strength.

\section{ACKNOWLEDGMENTS}

This work was performed at the Swiss Muon Source $(\mathrm{S} \mu \mathrm{S})$, Paul Scherrer Institut (PSI), Switzerland. We acknowledge financial support by the Swiss National Science Foundation.

\section{APPENDIX A: LONDON MODEL IN THE THIN-FILM LIMIT}

The calculation of the second moment of the $z$ component of the magnetic induction in the vortex state of a strong type-II superconductor within the London framework will

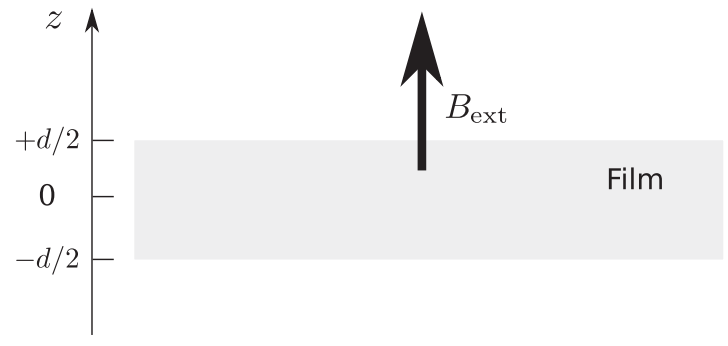

FIG. 9. Sketch of the thin-film geometry assumed for the calculation.

be presented. It is a straightforward extension of Ref. [52]. Figure 9 depicts the geometry used in the calculation.

It is assumed that the vortex lattice is not altered geometrically compared to the bulk, and hence the $x-y$ components of the calculation will be equal to the bulk. Furthermore, since we are interested in the $\mu$ SR line broadening due to the vortex lattice in the aforementioned geometry, only $B_{z}(x, y, z)$ needs to be calculated. Fortunately, this is exactly the component which is continuous at the boundary film/vacuum (see Ref. [53]).

In the film the London equation takes the form

$$
\mathbf{B}-\lambda^{2} \Delta \mathbf{B}=\Phi_{0} \sum_{n} \delta\left(\mathbf{r}-\mathbf{r}_{n}\right) \hat{\mathbf{z}} .
$$

Since the problem is periodic in the $x-y$ plane, the following ansatz is chosen:

$$
B_{z}(\mathbf{r}, z)=\sum_{\mathbf{k}} b_{z}(\mathbf{k}, z) \exp (+i \mathbf{k} \cdot \mathbf{r}),
$$

with $\mathbf{r}=(x, y)$ and correspondingly $\mathbf{k}=\left(k_{x}, k_{y}\right)$. Using this ansatz, the left side of Eq. (A1) will read as

$$
\begin{aligned}
\frac{d^{2}}{d z^{2}} B_{z} & =\sum_{\mathbf{k}} \frac{d^{2} b_{z}}{d z^{2}}(\mathbf{k}, z) \exp (+i \mathbf{k} \cdot \mathbf{r}), \\
\frac{d^{2}}{d(x, y)^{2}} B_{z} & =\sum_{\mathbf{k}} b_{z}(\mathbf{k}, z)\left(-k_{(x, y)}^{2}\right) \exp (+i \mathbf{k} \cdot \mathbf{r}) .
\end{aligned}
$$

Taking furthermore into account the equality

$$
\Phi_{0} \sum_{n} \delta\left(\mathbf{r}-\mathbf{r}_{n}\right) \hat{\mathbf{z}}=\underbrace{N \Phi_{0}}_{=: \bar{B}} \sum_{\mathbf{K}} \exp (+i \mathbf{K} \cdot \mathbf{r}),
$$

Eq. (A1) reads as

$$
\begin{aligned}
& \sum_{\mathbf{k}}\left\{b_{z}-\lambda^{2} \frac{d^{2} b_{z}}{d z^{2}}+\lambda^{2}\left(k_{x}^{2}+k_{y}^{2}\right) b_{z}\right\} \\
& \times \exp (+i \mathbf{k} \cdot \mathbf{r})=\bar{B} \sum_{\mathbf{K}} \exp (+i \mathbf{K} \cdot \mathbf{r}),
\end{aligned}
$$

and since $\exp (+i \mathbf{k} \cdot \mathbf{r})$ form a fully orthogonal set of functions, Eq. (A4) reduces to

$$
\bar{B}=b_{z}\left[1+\lambda^{2}\left(K_{x}^{2}+K_{y}^{2}\right)\right]-\lambda^{2} \frac{d^{2} b_{z}}{d z^{2}} .
$$

Equation (A5) is the differential equation to be solved in the film. Outside the film, the Laplace equation holds:

$$
\Delta B_{z}(x, y, z)=0,
$$


and hence using again the ansatz (A2), it follows

$$
\frac{d^{2} b_{z}}{d z^{2}}=\left(K_{x}^{2}+K_{y}^{2}\right) b_{z} .
$$

To solve Eq. (A5), the particular solution

$$
b_{z}^{\mathrm{p}}=\frac{\bar{B}}{\beta^{2}}
$$

is used, where $\beta^{2}=1+\lambda^{2}\left(K_{x}^{2}+K_{y}^{2}\right)$. The homogeneous solution is obtained via the ansatz

$$
b_{z}^{\mathrm{h}}=c_{1} \exp (+\alpha z)+c_{2} \exp (-\alpha z) .
$$

Setting Eq. (A9) into (A5) leads to

$$
\alpha=\frac{\beta}{\lambda}
$$

Therefore, the solution of Eq. (A4) will have the form

$$
b_{z}^{\mathrm{F}}=b_{z}^{\mathrm{p}}+b_{z}^{\mathrm{h}}=\frac{\bar{B}}{\beta^{2}}+c_{1} \exp (+\alpha z)+c_{2} \exp (-\alpha z),
$$

where $c_{1,2}$ will be determined via the boundary conditions. For the vacuum side where the Laplace equation holds, the $z$-component ansatz is

$$
b_{z}^{\mathrm{V}}= \begin{cases}c_{+} \exp (-\alpha z), & z \geqslant+d / 2 \\ c_{-} \exp (+\alpha z), & z \leqslant-d / 2 .\end{cases}
$$

The boundary conditions are

$$
\begin{aligned}
& b_{z}^{\mathrm{F}}(z= \pm d / 2)=b_{z}^{\mathrm{V}}( \pm d / 2), \\
& \frac{b_{z}^{\mathrm{F}}}{d z}(z= \pm d / 2)=\frac{b_{z}^{\mathrm{V}}}{d z}( \pm d / 2) .
\end{aligned}
$$

Using these boundary conditions, $c_{1}, c_{2}, c_{+}$, and $c_{-}$can be calculated, which leads to

$$
\begin{aligned}
z \geqslant+d / 2: b_{z}(\mathbf{K}, z)=\frac{\bar{B}}{\beta^{2}} \exp \left(-\frac{z \beta}{\lambda}\right) \sinh \left(\frac{\beta d}{2 \lambda}\right) \\
z \leqslant-d / 2: b_{z}(\mathbf{K}, z)=\frac{\bar{B}}{\beta^{2}} \exp \left(+\frac{z \beta}{\lambda}\right) \sinh \left(\frac{\beta d}{2 \lambda}\right), \\
-d / 2 \leqslant z \leqslant+d / 2: b_{z}(\mathbf{K}, z) \\
=\frac{\bar{B}}{\beta^{2}}\left[1-\cosh \left(\frac{\beta z}{\lambda}\right) \exp \left(-\frac{\beta d}{2 \lambda}\right)\right]
\end{aligned}
$$

with

$$
\beta^{2}=1+\lambda^{2}\left(K_{x}^{2}+K_{y}^{2}\right)=1+\lambda^{2}|\mathbf{K}|^{2} .
$$

The $\mu$ SR line width of the vortex lattice can be approximated by the second moment of the field distribution along the $z$ component. The second moment is

$$
\left\langle\Delta B_{z}^{2}\right\rangle=\left\langle B_{z}^{2}\right\rangle-\left\langle B_{z}\right\rangle^{2}
$$

and, therefore, using Eq. (A2) for a given $z$ value, it follows

$$
\left\langle\Delta B_{z}^{2}\right\rangle(z)=\sum_{\mathbf{K} \neq 0}\left|b_{z}(\mathbf{K}, z)\right|^{2} .
$$
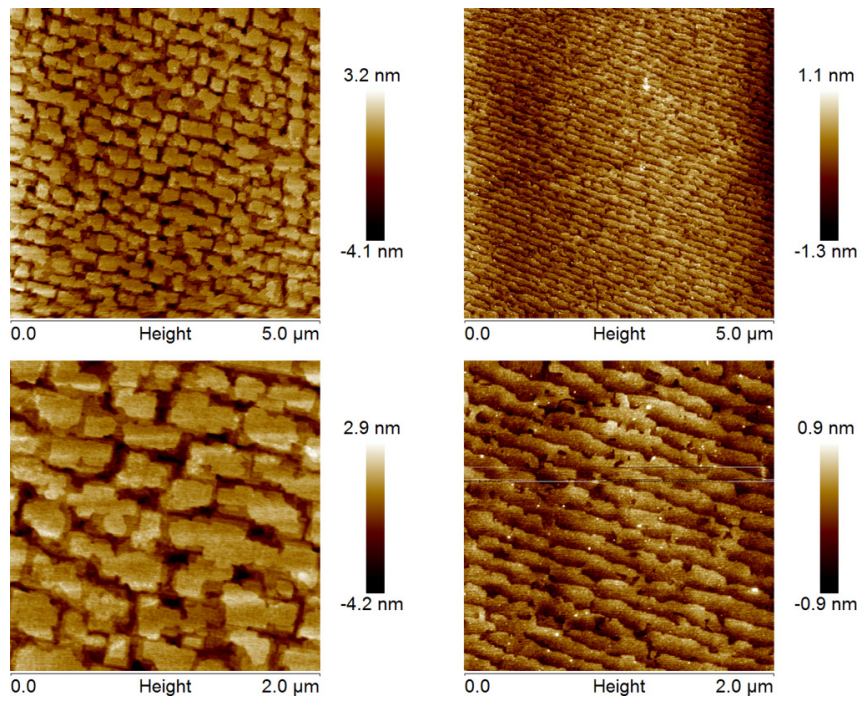

FIG. 10. First (second) row $5 \times 5 \mu \mathrm{m}^{2}\left(2 \times 2 \mu \mathrm{m}^{2}\right)$ atomic force microscopy (AFM) images of two typical thin films: a 40-nm-thick LSCO film grown on LSAO (left) and a 40-nm-thick LSCO film grown on STO (right).

Taking into account the perfect triangular lattice where ${ }^{1}$

$$
\mathbf{K}^{2}=K_{m, n}^{2}=\frac{16 \pi^{2}}{3 d^{2}}\left(m^{2}-m n+n^{2}\right) \quad \forall(m, n) \neq(0,0)
$$

and

$$
d^{2}=\frac{2 \Phi_{0}}{\bar{B} \sqrt{3}},
$$

the width measured by a low-energy $\mu \mathrm{SR}$ experiment can numerically evaluated as

$$
\begin{aligned}
\sigma_{\mathrm{sc}}^{2} & =\gamma_{\mu}^{2} \int_{-\infty}^{+\infty}\left\langle\Delta B_{z}^{2}\right\rangle(z) n(z, E) d z \\
& =\gamma_{\mu}^{2} \int_{-\infty}^{+\infty} \sum_{(m, n) \neq(0,0)}\left|b_{z}(\mathbf{K}, z)\right|^{2} n(z, E) d z,
\end{aligned}
$$

where $n(z, E)$ is the muon stopping distribution.

\section{APPENDIX B: THIN FILMS' CHARACTERIZATION}

The $\mathrm{La}_{1.84} \mathrm{Sr}_{0.16} \mathrm{CuO}_{4}$ epitaxial films were synthesized using an atomic layer-by-layer oxide molecular beam epitaxy (ALL-oxide-MBE) system. Single-crystal $\mathrm{LaSrAlO}_{4}$ (LSAO) and $\mathrm{SrTiO}_{3}$ (STO) substrates, $10 \times 10 \times 1 \mathrm{~mm}^{3}$ in size, epipolished with surface perpendicular to the [001] crystal axis were used. The film growth and quality were monitored in real time using reflection high-energy electron diffraction (RHEED) system, and tested subsequently by atomic force microscopy (AFM) as well as by susceptibility and x-ray diffraction measurements.

\footnotetext{
${ }^{1} \mathrm{Be}$ aware that the definition of Eq. (A20) is different compared to what is found in Ref. [52]. Here, the $m, n$ run over all positive and negative numbers!
} 

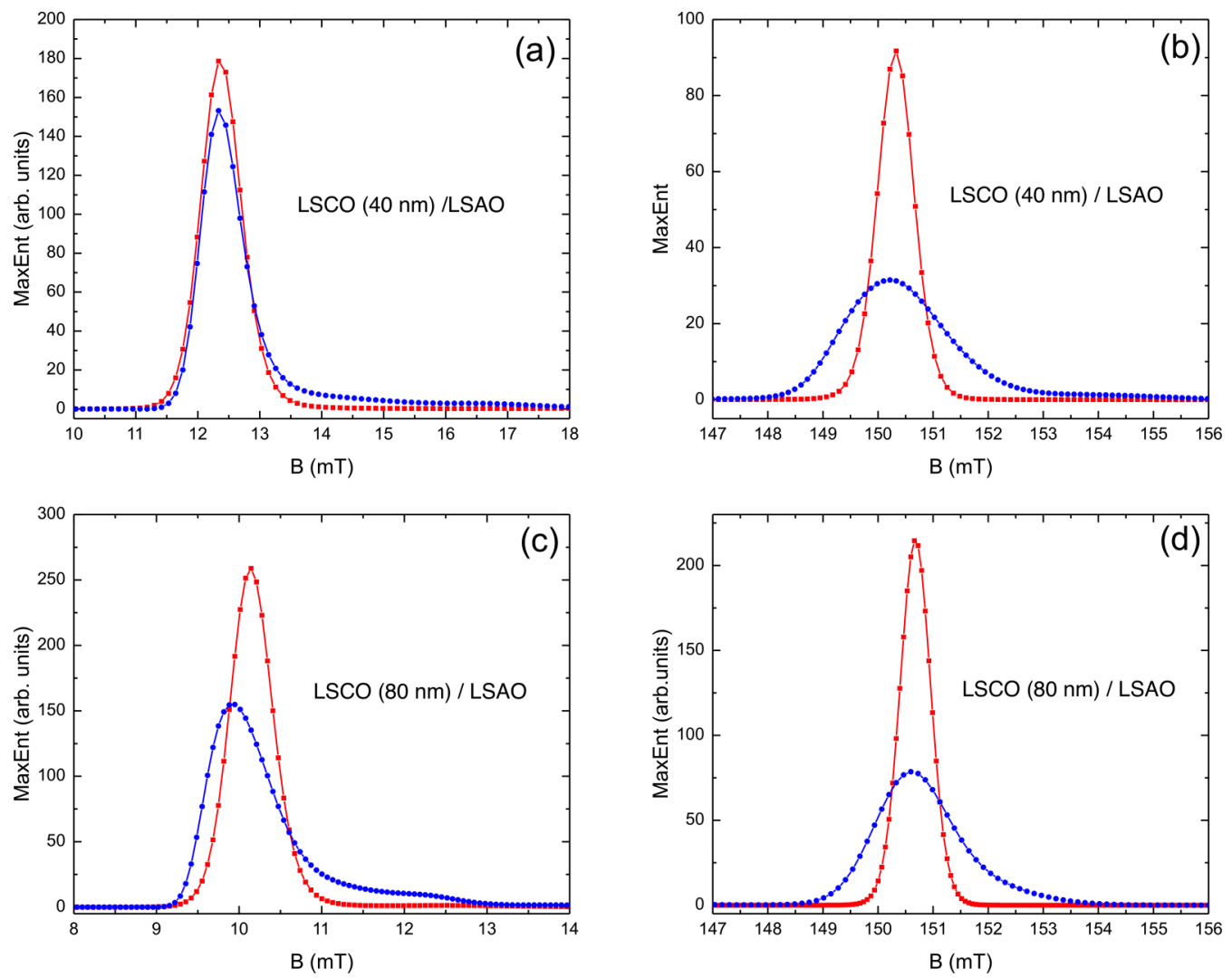

FIG. 11. Representative Fourier transform of the muon polarization function $p(t)$, using the maximum entropy technique. The red lines correspond to a sample in the normal phase at a temperature of $50 \mathrm{~K}$ while the sample is in the superconducting phase ( $T=4 \mathrm{~K}$ ) for the blue lines. The film thickness is $40 \mathrm{~nm}$ in (a) and (b) and respectively $80 \mathrm{~nm}$ for (c) and (d). The externally applied magnetic field is weak $(10-12 \mathrm{mT})$ in (a) and (c) and stronger $(\sim 150 \mathrm{mT})$ for (b) and (d). A vertical comparison of the graphs allows a direct visualization of the crossover in the field dependence of the magnetic field broadening (see text).

In Fig. 10, typical AFM images are presented. Two types of growth can be identified. When the substrate strain is fully relaxed, as in the the case of a 40-nm LSCO film on a STO substrate, the growth occurs layer by layer and the atomic steps visible on the AFM images match the substrate roughness. On the other hand, when the LSCO film is under compressive strain, such as in the the case of a 40-nm LSCO film on a LSAO substrate, a columnar type of growth is favored on the first $7 \mathrm{~nm}$ from the vacuum interface. This can be intuitively understood as the columnar structure allows a lower-energy state with some strain being released. At a certain depth, however, the surface energy loss is greater than this gain. Note that these $7 \mathrm{~nm}$ correspond to the superconducting "dead layer" found with the fit of the magnetic depth profile with the numerical London model.

\section{APPENDIX C: MAGNETIC FIELD DISTRIBUTION IN THE FILMS IN THE SUPERCONDUCTING AND NORMAL PHASES}

The magnetic field broadening due to the vortex lattice in the superconducting mixed state was determined using low-energy muon spin rotation (LE- $\mu \mathrm{SR})$. The Fourier transform of the muon polarization function $p(t)$ is a direct measure of the magnetic field broadening, in the direction perpendicular to the positrons detectors, in the sample, at the muon stopping site. In our case, we measure the field broadening along the [001] direction. Representative Fourier transform, obtained using the maximum entropy technique, is presented in Fig. 11. The magnetic field distribution observed at high temperatures ( $T=50 \mathrm{~K}$, blue lines in the figures) in the normal state is due to the nuclear spin contribution. This contribution can vary between samples and upon application of an external magnetic field, due to slight variation in the muon stopping site, the atomic surrounding of the muon, and the polarization of the nuclear spins. At low temperatures $(T=5 \mathrm{~K}$, red lines in the figures), the modification of the magnetic field broadening, in comparison to the same sample under the same magnetic field at high temperature, is mainly due to the superconducting vortex lattice.

In Fig. 11 one sees clearly that the superconducting vortex lattice contribution to the magnetic field broadening is for both samples larger upon larger applied magnetic field (figures on the right column). The skewness appears to be stronger at lower magnetic fields. We believe, however, that this is an artifact due to vortex pinning, as the distance between vortices is large upon lower external magnetic field and therefore the forces acting upon the individual vortices in order to form the vortex lattice smaller. 
The crossover discussed in the main part of the paper is directly visible upon comparison of the figures in the same row in Fig. 11. In the first column (lower applied external magnetic field), the superconducting vortex lattice contribution to the magnetic field broadening is clearly bigger in the lower figure or thicker film (80 $\mathrm{nm}$ ), while in the second column the top figure, thinner film (40 $\mathrm{nm})$ as a slightly stronger superconducting vortex lattice contribution. The absence of any visible skewness is here expected as the effective magnetic penetration depth is large.
[1] J. Bednorz and K. Müller, Z. Phys. B: Condens. Matter 64, 189 (1986).

[2] H. Sato, A. Tsukada, M. Naito, and A. Matsuda, Phys. Rev. B 61, 12447 (2000).

[3] D. Erskine, E. Hess, P. Y. Yu, and A. M. Stacy, J. Mater. Res. 2, 783 (1987).

[4] F. Nakamura, J. Hori, T. Goko, Y. Uno, N. Kikugawa, and T. Fujita, J. Low Temp. Phys. 117, 1145 (1999).

[5] H. Sato and M. Naito, Phys. C (Amsterdam) 274, 221 (1997).

[6] Indeed, in the simplest form of BCS formalism $k_{B} T_{c}=1.13 E_{D}$ $\exp (-1 /[N(0) V])$ with $k_{B}$ the Boltzmann constant, $E_{D}$ the Debye cutoff energy, $N(0)$ the density of states, and $V$ the electron-phonon coupling potential [54]. The exact formulation of this relation is modified in more complex theory or the ones involving a different coupling interaction. However, it is reasonable to assume an identical trend between the superconducting transition temperature and the two main superconducting parameters that are the density of states or carrier concentration and the coupling interaction, namely, an increase of $T_{c}$ with the increase of $N(0)$ or $V$. In addition, one expects $n_{S} \propto N(0)$ and a direct relation between $m^{\star}$ and $V$ sometimes given as $m^{\star} \propto(1+V)$ [55]. Therefore, using Eq. (1), an increase of $T_{c}$ due to an increase in $N(0)(V)$ will lead to a decrease (respectively an increase) of $\lambda$.

[7] B. Josephson, Phys. Lett. 1, 251 (1962).

[8] J. M. Imer, F. Patthey, B. Dardel, W. D. Schneider, Y. Baer, Y. Petroff, and A. Zettl, Phys. Rev. Lett. 62, 336 (1989).

[9] J.-P. Locquet, J. Perret, J. Fompeyrine, E. Machler, J. W. Seo, and G. Van Tendeloo, Nature (London) 394, 453 (1998).

[10] W. Si, H.-C. Li, and X. X. Xi, Appl. Phys. Lett. 74, 2839 (1999).

[11] S. Trommler, P. Pahlke, R. Hühne, L. Schultz, and B. Holzapfel, J. Phys.: Conf. Ser. 234, 012045 (2010).

[12] E. Morenzoni, T. Prokscha, A. Suter, H. Luetkens, and R. Khasanov, J. Phys.: Condens. Matter 16, S4583 (2004).

[13] J. E. Sonier, J. H. Brewer, and R. F. Kiefl, Rev. Mod. Phys. 72, 769 (2000)

[14] C. Niedermayer, E. M. Forgan, H. Glückler, A. Hofer, E. Morenzoni, M. Pleines, T. Prokscha, T. M. Riseman, M. Birke, T. J. Jackson et al., Phys. Rev. Lett. 83, 3932 (1999).

[15] F. Baiutti, G. Christiani, and G. Logvenov, Beilstein J. Nanotechnol. 5, 596 (2014).

[16] R. Khasanov, A. Shengelaya, A. Maisuradze, F. LaMattina, A. Bussmann-Holder, H. Keller, and K. A. Müller, Phys. Rev. Lett. 98, 057007 (2007).

[17] D. J. Werder, C. H. Chen, M. Gurvitch, B. Miller, L. F. Schneemeyer, and J. V. Waszczak, Physica C: Superconduct. 160, 411 (1989).

[18] T. Prokscha, E. Morenzoni, K. Deiters, F. Foroughi, D. George, R. Kobler, A. Suter, and V. Vrankovic, Nucl. Instrum. Methods Phys. Res., Sect. A 595, 317 (2008).

[19] A. Yaouanc and P. Dalmas de Réotier, Muon Spin Rotation, Relaxation, and Resonance: Applications to Condensed Mat- ter, International Series of Monographs on Physics (Oxford University Press, Oxford, 2011).

[20] D.R. Harshman, A. P. Mills, J. L. Beveridge, K. R. Kendall, G. D. Morris, M. Senba, J. B. Warren, A. S. Rupaal, and J. H. Turner, Phys. Rev. B 36, 8850 (1987).

[21] H. Saadaoui, Z. Salman, T. Prokscha, A. Suter, B. Wojek, and E. Morenzoni, Phys. Proc. 30, 164 (2012).

[22] A. Suter and B. Wojek, Phys. Proc. 30, 69 (2012).

[23] C. Cancellieri, The Growth by Laser Ablation and Electronic Properties of Thin Cuprate Films: $\mathrm{Bi}_{2} \mathrm{Sr}_{2-x} \mathrm{La}_{x} \mathrm{CuO}_{6}$ and $\mathrm{La}_{2-x} \mathrm{Sr}_{x} \mathrm{CuO}_{4}$, Ph.D. thesis, Ecole Polytechnique Federale de Lausanne, 2008.

[24] R. People and J. C. Bean, Appl. Phys. Lett. 47, 322 (1985).

[25] X. Qiu, High-Temperature Superconductors, Woodhead Publishing Series in Electronic and Optical Materials (Elsevier. Amsterdam, 2011).

[26] F. Nakamura, T. Goko, J. Hori, Y. Uno, N. Kikugawa, and T. Fujita, Phys. Rev. B 61, 107 (2000).

[27] I. Bozovic, G. Logvenov, I. Belca, B. Narimbetov, and I. Sveklo, Phys. Rev. Lett. 89, 107001 (2002).

[28] J. H. van der Merwe and W. A. Jesser, J. Appl. Phys. 64, 4968 (1988).

[29] C. Niedermayer, C. Bernhard, T. Blasius, A. Golnik, A. Moodenbaugh, and J. I. Budnick, Phys. Rev. Lett. 80, 3843 (1998).

[30] E. Stilp, A. Suter, T. Prokscha, E. Morenzoni, H. Keller, B. M. Wojek, H. Luetkens, A. Gozar, G. Logvenov, and I. Božović, Phys. Rev. B 88, 064419 (2013).

[31] Y. J. Uemura, T. Yamazaki, D. R. Harshman, M. Senba, and E. J. Ansaldo, Phys. Rev. B 31, 546 (1985).

[32] J. Biersack and W. Eckstein, Appl. Phys. A 34, 73 (1984).

[33] E. H. Brandt, Phys. Rev. Lett. 78, 2208 (1997).

[34] E. H. Brandt, Phys. Rev. B 68, 054506 (2003).

[35] A. I. Gubin, K. S. Il'in, S. A. Vitusevich, M. Siegel, and N. Klein, Phys. Rev. B 72, 064503 (2005).

[36] Y. Wang and H.-H. Wen, Europhys. Lett. 81, 57007 (2008).

[37] T. J. Jackson, T. M. Riseman, E. M. Forgan, H. Glückler, T. Prokscha, E. Morenzoni, M. Pleines, C. Niedermayer, G. Schatz, H. Luetkens, and J. Litterst, Phys. Rev. Lett. 84, 4958 (2000).

[38] M. Lindstrom, B. Wetton, and R. Kiefl, J. Eng. Math. 85, 149 (2014).

[39] B. Lake, G. Aeppli, K. N. Clausen, D. F. McMorrow, K. Lefmann, N. E. Hussey, N. Mangkorntong, M. Nohara, H. Takagi, T. E. Mason, and A. Schröder, Science 291, 1759 (2001).

[40] G. M. De Luca, G. Ghiringhelli, M. Moretti Sala, S. Di Matteo, M. W. Haverkort, H. Berger, V. Bisogni, J. C. Cezar, N. B. Brookes, and M. Salluzzo, Phys. Rev. B 82, 214504 (2010).

[41] G. Seyfarth, J. P. Brison, M.-A. Méasson, J. Flouquet, K. Izawa, Y. Matsuda, H. Sugawara, and H. Sato, Phys. Rev. Lett. 95, 107004 (2005)

[42] L. Howald, M. Taupin, and D. Aoki, Phys. Res. Int. 2014, 454939 (2014). 
[43] Z. Shermadini, J. Kanter, C. Baines, M. Bendele, Z. Bukowski, R. Khasanov, H.-H. Klauss, H. Luetkens, H. Maeter, G. Pascua et al., Phys. Rev. B 82, 144527 (2010).

[44] F. Brückner, R. Sarkar, M. Günther, H. Kühne, H. Luetkens, T. Neupert, A. P. Reyes, P. L. Kuhns, P. K. Biswas, T. Stürzer et al., Phys. Rev. B 90, 220503 (2014).

[45] J. K. Dong, T. Y. Guan, S. Y. Zhou, X. Qiu, L. Ding, C. Zhang, U. Patel, Z. L. Xiao, and S. Y. Li, Phys. Rev. B 80, 024518 (2009).

[46] R. Khasanov, A. Shengelaya, J. Karpinski, A. BussmannHolder, H. Keller, and K. Müller, J. Supercond. Novel Magn. 21, 81 (2008).

[47] Y. Ponomarev, V. Alyoshin, E. Antipov, T. Oskina, A. Krapf, S. Kulbachinskii, M. Mikheev, M. Sudakova, S. Tchesnokov, and L. Fisher, JETP Lett. 100, 126 (2014).
[48] A. Damascelli, Z. Hussain, and Z.-X. Shen, Rev. Mod. Phys. 75, 473 (2003).

[49] D. LeBoeuf, N. Doiron-Leyraud, B. Vignolle, M. Sutherland, B. J. Ramshaw, J. Levallois, R. Daou, F. Laliberté, O. CyrChoinière, J. Chang et al., Phys. Rev. B 83, 054506 (2011).

[50] S. E. Suchitra, N. Harrison, E. Palm, T. P. Murphy, C. H. Mielke, R. Liang, D. A. Bonn, W. N. Hardy, and G. G. Lonzarich, Nature (London) 454, 200 (2008).

[51] E. H. Brandt, Phys. Rev. B 71, 014521 (2005).

[52] E. H. Brandt, Phys. Rev. B 37, 2349 (1988).

[53] J. Jackson, Classical Electrodynamics (Wiley, New York, 1975).

[54] J. Bardeen, L. N. Cooper, and J. R. Schrieffer, Phys. Rev. 108, 1175 (1957).

[55] G. W. Crabtree, D. H. Dye, D. P. Karim, S. A. Campbell, and J. B. Ketterson, Phys. Rev. B 35, 1728 (1987). 